\title{
Bloqueos de tronco
}

\section{Truncal blocks}

Sebastián Layera MD. ${ }^{1}$, Daniela Bravo MD. ${ }^{1}$, Julián Aliste MD. ${ }^{1}$

\begin{abstract}
Truncal blocks have recently been positioned as an alternative to neuraxial analgesia. The injection of local anesthetics in interfascial planes was initially guided by anatomical references, to later evolve towards a more selective administration when guided by ultrasound. Successful execution of truncal blocks requires detailed knowledge of the chest and abdominal walls anatomy. The same logic allows us to understand its potential benefits concerning perioperative analgesia, as well as its limitations and therapeutic margins. Secondary to a growing interest in less invasive techniques and analgesic techniques with a more favorable risk-benefit profile, the available evidence in this field is in continuous development. Thus, in the present review, the technical aspects of these blocks will be evaluated, emphasizing the sonoanatomy, and assessing the best evidence to support the use of each technique.

Término Mesh: nerve block, regional anesthesia, local anesthetics, fascia.
\end{abstract}

\section{RESUMEN}

Los bloqueos de tronco se han posicionado recientemente como una alternativa frente a la analgesia neuroaxial. La inyección de anestésicos locales en planos interfasciales inicialmente fue guiado por referencias anatómicas, para posteriormente evolucionar hacia una administración más selectiva al ser guiada por el ultrasonido. La ejecución exitosa de los bloqueos de tronco requiere un conocimiento detallado de la anatomía de las paredes del tórax y abdomen. Esta misma lógica nos permite entender sus potenciales beneficios en relación con la analgesia perioperatoria, como también sus limitaciones y margen terapéutico. La evidencia disponible está en continuo desarrollo, dado el creciente interés que concitan técnicas menos invasivas y con un perfil de riesgobeneficio potencialmente más favorable. En la presente revisión se evaluarán los aspectos técnicos de cada bloqueo, poniendo énfasis en la sonoanatomía, y evaluando la mejor evidencia que sustente el uso de cada técnica.

\section{Key words:}

Nerve block, regional anesthesia, local anesthetics, fascia

\section{Palabras clave:}

Bloqueos regionales, anatomía regional, anestesia local, fascia

\footnotetext{
Departamento de Anestesiología y Medicina Perioperatoria, Universidad de Chile.
}

Fecha de recepción: 18 de noviembre de 2019

Fecha de aceptación: 18 de noviembre de 2019

\section{ORCID}

https://orcid.org/0000-0001-5690-9609

\section{Correspondencia:}

Sebastián Layera

Email: sebastianlayera@gmail.com 


\section{Introducción}

E I I uso de una técnica neuroaxial para el manejo del dolor postoperatorio, tanto en cirugía torácica como abdominal mayor, constituye un pilar fundamental en nuestra práctica clínica como anestesiólogos. Sin embargo, el bloqueo epidural torácico está considerado como uno de los procedimientos con mayor dificultad técnica[1], tiene una alta tasa de falla primaria en centros docentes[2] y se asocia a diversas complicaciones derivadas del procedimiento (ej. punción dural accidental, hematoma o infección epidural) y de los fármacos que se utilizan en forma continua (ej. hipotensión, náusea y vómitos, retención urinaria).

Con el propósito de tener alternativas a la analgesia epidural torácica (AET) han surgido recientemente una amplia variedad de técnicas de anestesia regional, cuyo objetivo primario es alejarse del neuroeje para evitar las complicaciones ya descritas, intentando al mismo tiempo lograr una adecuada analgesia postoperatoria.

Los bloqueos de tronco tienen como característica común la búsqueda de espacios anatómicos interfasciales o subfasciales, que permitan la administración de anestésicos locales, lo que muchas veces debe ser en grandes volúmenes, con el objetivo de bloquear nervios periféricos por difusión del fármaco dentro de dicho espacio. De esta lógica se desprenden algunos de sus riesgos y limitaciones, toda vez que existe un mayor riesgo de toxicidad sistémica por anestésicos locales por la masa de droga requerida (ej. bloqueos bilaterales, amplias áreas de absorción)[3], además de la inherente dificultad procedimental para ubicar con precisión espacios anatómicos virtuales.

El conocimiento de la inervación de la pared torácica y abdominal, y su exploración en tiempo real con el ultrasonido (US), han permitido durante las últimas décadas el desarrollo de nuevas técnicas de anestesia regional, además del refinamiento de otras que inicialmente fueron efectuadas por referencias anatómicas.

\section{Inervación del tronco}

La inervación de la pared del tronco depende fundamentalmente de los nervios torácicos espinales. Éstos se dividen en un ramo posterior y anterior luego de su emergencia del agujero intervertebral[4]. El ramo posterior inerva músculos, articulaciones y piel de la parte dorsal del tórax y abdomen, mientras el ramo anterior inerva la contraparte ventral, incluyen- do el recubrimiento pleuro-peritoneal de ambas cavidades. Los ramos anteriores de los nervios espinales torácicos se denominan nervios intercostales, debido a su recorrido en el espacio intercostal. Cada nervio (excepto T1 que contribuye a la inervación de la extremidad superior) a nivel de la línea axilar media da un ramo cutáneo lateral que atraviesa los músculos intercostales, dividiéndose a su vez en un ramo anterior y posterior que inervan la piel de la pared torácica antero-lateral. El ramo cutáneo anterior corresponde al ramo terminal de los nervios intercostales segundo al sexto y emerge entre los músculos intercostales cerca del borde lateral del esternón, dando un ramo medial y otro lateral que inervan la piel de la línea media torácica.

Los nervios intercostales de T6 a T11 tienen un trayecto inicialmente intercostal, sin embargo, en su trayecto hacia la línea media envían ramos hacia la pared abdominal, por lo cual reciben el nombre de nervios tóracoabdominales[5]. Son responsables de la inervación de los músculos de la pared abdominal anterior, además de proveer la sensibilidad cutánea bajo el reborde costal entre la apófisis xifoides y el ombligo. El nervio subcostal (T12) no tiene trayecto intercostal, y transcurre entre los músculos de la pared abdominal, inervando músculos y la piel sobre la sínfisis del pubis.

Los nervios ilioinguinal e iliohipogástrico, originados en T12-L1 y L1 respectivamente, son ramas terminales del plexo lumbar, no obstante, contribuyen en la inervación de la pared abdominal inferior y de la zona inguinal. Además de la inervación cutánea y muscular contribuyen en la sensibilidad del peritoneo parietal.

Los nervios que contribuyen a la inervación de la pared abdominal anterior (tóraco-abdominales, subcostales y primer lumbar) tienen un recorrido común en el plano intermuscular entre el músculo transverso del abdomen (MTA) y el músculo oblicuo interno (MOI), plano conocido como TAP en inglés (Figura 2). El lugar exacto donde cada nervio ingresa al TAP determina en parte la ubicación óptima para lograr su bloqueo exitoso. Los nervios de T6 a T9 ingresan al TAP medial a la línea axilar anterior, mientras los nervios de T10 a L1 lo hacen desde una posición más lateral[6].

Estudios anatómicos cadavéricos han demostrado que no existe una disposición metamérica estricta de inervación, ya que los nervios se ramifican y comunican entre sí en diferentes niveles. Así, los nervios segmentarios de T9 a L1 forman a nivel de la rama ascendente de la arteria circunfleja ilíaca profunda un plexo longitudinal con disposición craneo-caudal, el 


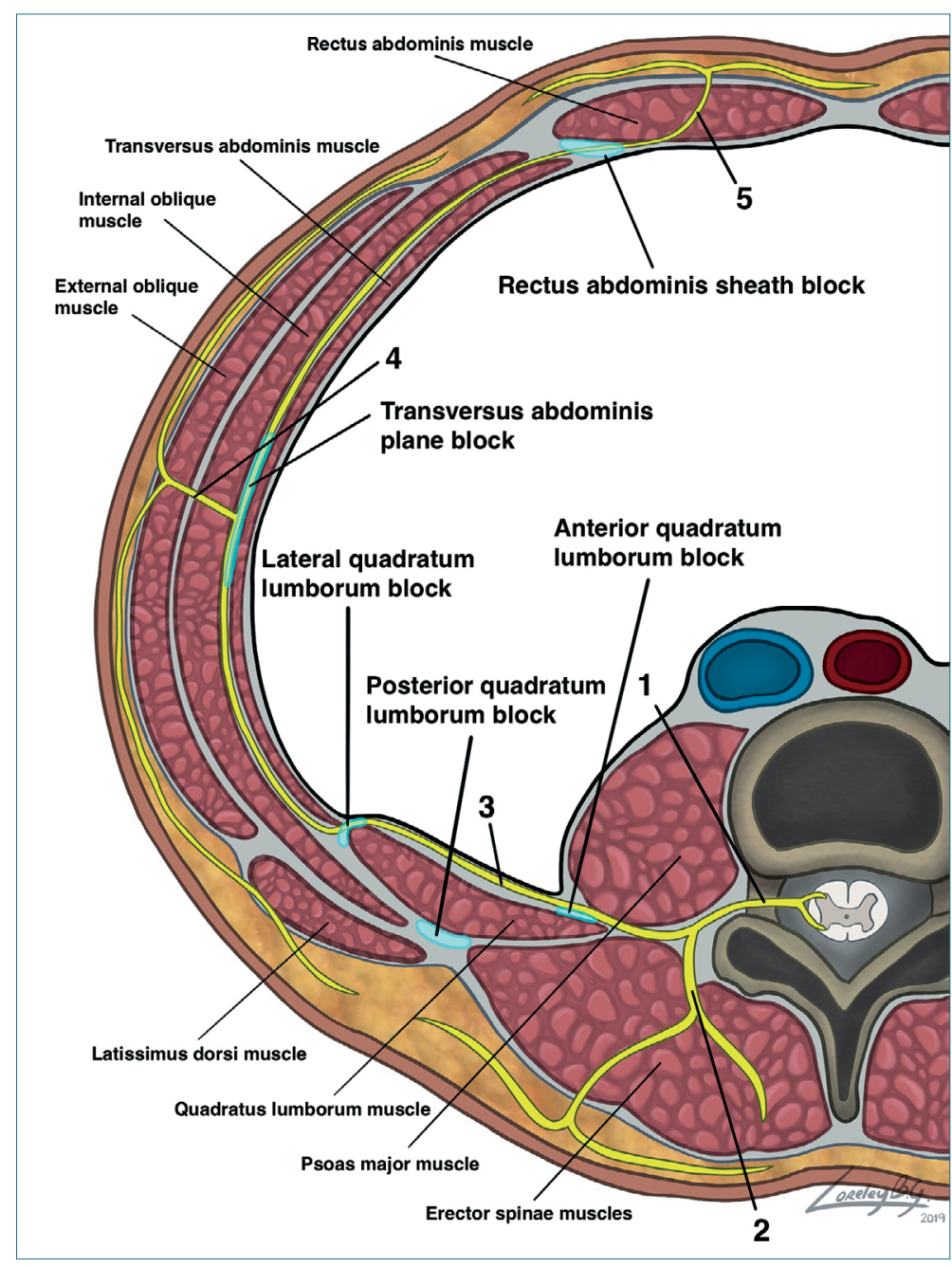

Figura 1. Anatomía de la pared torácica a nivel de T4 y sitios de inyección para bloqueos PECS, bloqueo plano del serrato y ESP. 1: nervio espinal, 2: ramo comunicante a cadena simpática, $\mathbf{3}$ : ramo posterior, 4: nervio interscostal, $\mathbf{5}$ : ramo cutáneo lateral, 6: ramo cutáneo anterior. cual se ha denominado 'plexo del TAP'. Posteriormente, los mismos nervios contribuyen a la formación del 'plexo de la vaina de los rectos', con similar dirección y en relación directa a la arteria epigástrica inferior profunda. Esto explica en parte la naturaleza multisegmentaria de la inervación de la pared abdominal y la línea media (T6-L1)[6].

La compleja inervación del tronco determina un gran desafío para el uso sistemático de nuevas técnicas de anestesia regional. Es también la explicación del efecto parcial de muchos bloqueos, y especialmente de aquellos que no logran bloquear los nervios espinales en su origen o en su trayecto. Esto se hace presente particularmente en cirugías que involucran línea media torácica o abdominal.

A continuación, se analizarán los bloqueos de tronco descritos a la fecha, en los cuales se dispone de evidencia proveniente de estudios clínicos randomizados de revistas con revisión de pares. Las conclusiones obtenidas sólo harán mención a la mejor evidencia existente a la fecha, obviando las series y reportes de casos, con la meta de realizar un análisis objetivo de los beneficios y limitaciones de cada técnica descrita.

\section{Bloqueos PECS y del plano del serrato}

Descrita inicialmente por Blanco en 2011[7], esta técnica guiada por US fue concebida como una alternativa a la analgesia peridural y el bloqueo paravertebral (BPV) en cirugía mamaria.

En su descripción original el bloqueo PECS I tiene 


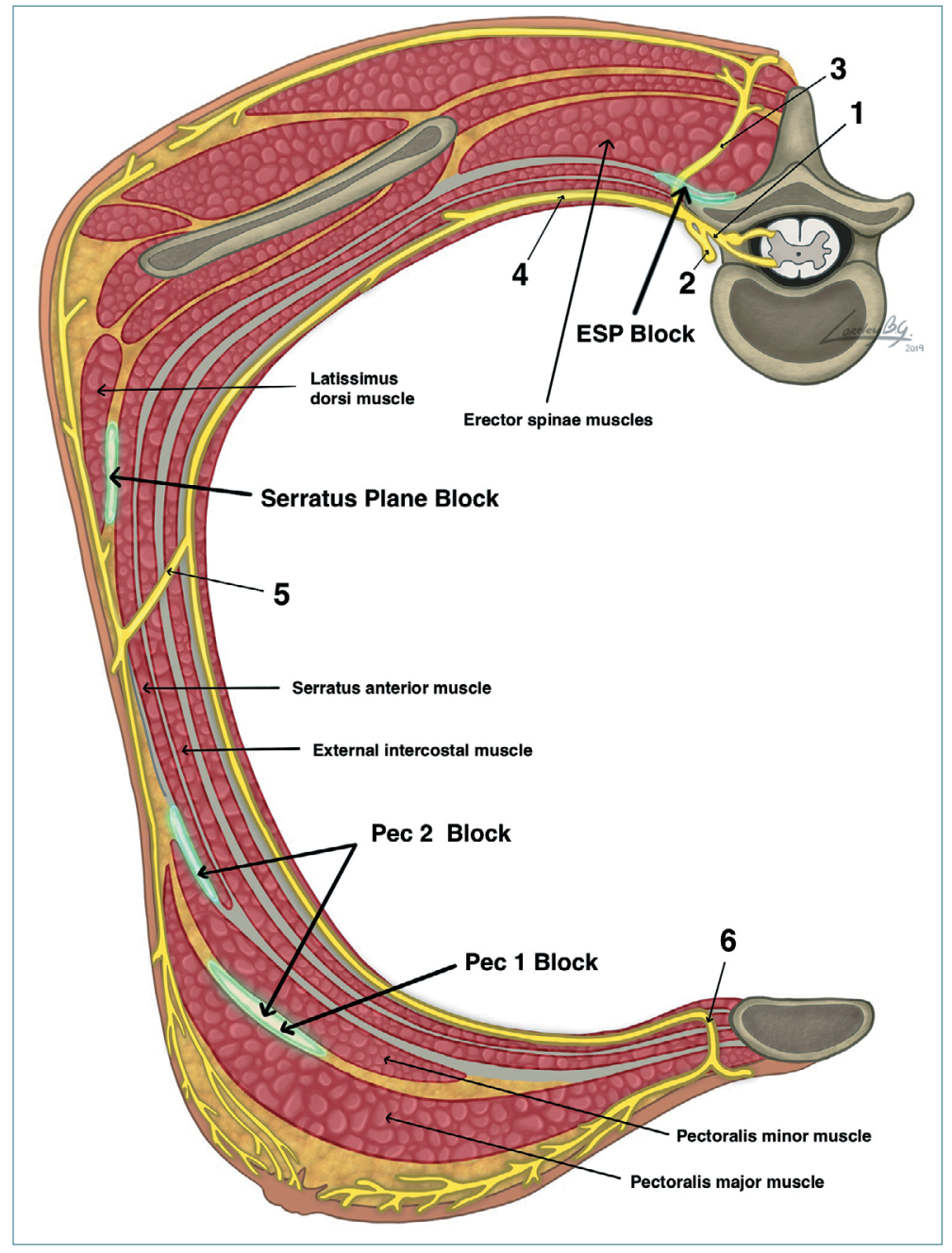

Figura 2. Anatomía de la pared abdominal a nivel de $L 4$ y sitios de inyección para bloqueos del cuadrado lumbar, TAP y vaina de los rectos. 1: nervio espinal, 2: ramo posterior, 3: ramo anterior, 4: ramo cutaneo lateral, 5: ramo cutaneo anterior. como objetivo administrar anestésico local $(\mathrm{AL})$ entre los músculos pectoral mayor (MPM) y pectoral menor (MPm) (Figura 1, 3A y 3B). El volumen sugerido para el bloqueo son 0,4 ml/kg de levobupivacaína 0,25\%, con lo cual el autor reportó mínimo uso de opioides posterior a cirugía mamaria, con resultados especialmente favorables en caso de prótesis o expansores mamarios[7]. El alivio del espasmo muscular se ha planteado como mecanismo analgésico, dado que los nervios pectorales sólo tendrían función motora. Sin embargo, a la fecha no existe evidencia que respalde esta teoría[8].

Posteriormente, el mismo autor presentó una modificación de la técnica, buscando una mejor cobertura analgésica en procedimientos más invasivos de mama y que incluyeran la axila. Esta técnica se deno- minó PECS II y consiste en un bloqueo con 2 sitios de inyección mediante una sola punción. En la primera inyección se administran $10 \mathrm{ml}$ de $\mathrm{AL}$ en el plano interfascial entre el MPM y el MPm. En la segunda, se inyectan $20 \mathrm{ml}$ de $\mathrm{AL}$ en el plano entre el MPm y el músculo serrato anterior (MSA) a nivel de la $2^{\text {da }}$ y $3^{\text {ra }}$ costilla (Figura 1, 3A y 3B). En esta ubicación el mecanismo analgésico estaría dado por el bloqueo del ramo cutáneo lateral de los nervios intercostales (T2 a T6), así como también de los nervios torácico largo y toracodorsal[9].

El bloqueo del plano del serrato (BPS) fue el último replanteamiento de los bloqueos PECS, buscando un bloqueo más selectivo de los nervios intercostales para lograr analgesia de la pared torácica antero-lateral. En esta variación se ubica el transductor en un 


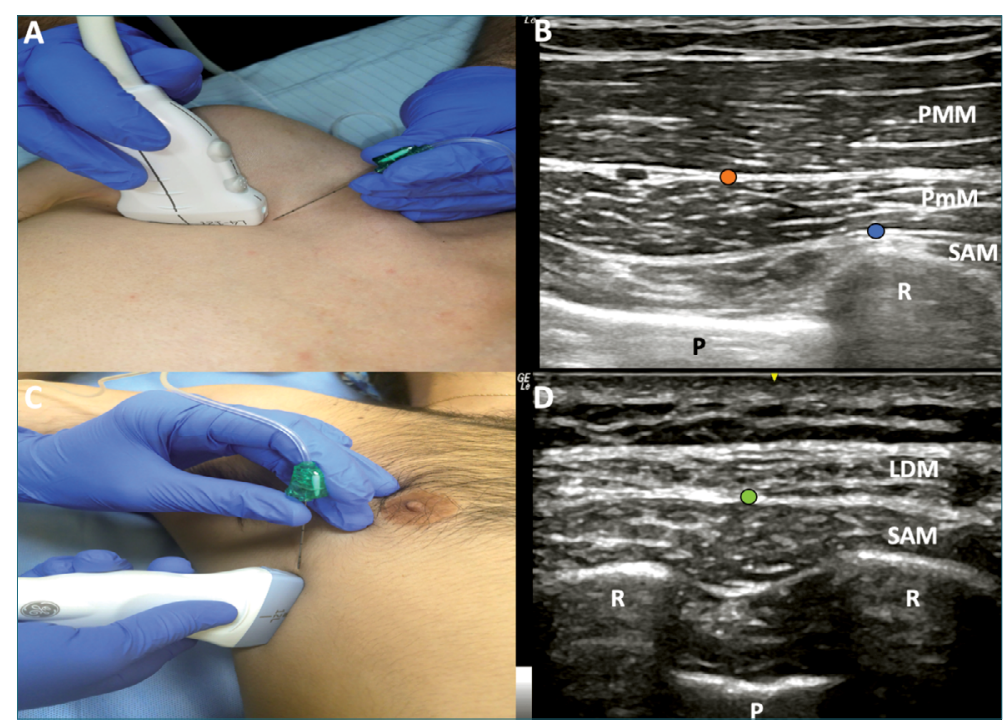

Figura 3: Bloqueos PECS y del plano del serrato. A: ubicación transductor lineal para bloqueos PECS I y II. B: sonoanatomía bloqueos PECS. C: ubicación transductor lineal para BPS. D: sonoanatomía bloqueo del plano del serrato. $C=$ costilla, MDA = músculo dorsal ancho, $\mathrm{MPM}=$ músculo pectoral mayor, $\mathrm{MPm}$ = músculo pectoral menor, MSA = músculo serrato anterior, $P=$ pleura, punto naranja = sitio inyección PECS 1 , punto azul = sitio segunda inyección PECS 2 , punto verde $=$ sitio inyección bloqueo del plano del serrato. plano sagital a nivel medio-clavicular. Una vez identificada la $2^{\text {da }}$ costilla se desplaza el transductor hacia la línea axilar media sobre la $5^{\text {ta }}$ costilla, en esa ubicación se observa de superficial a profundo los músculos latísimo del dorso y MSA (Figura 1, 3C y 3D). La inyección de AL puede realizarse tanto superficial como profundo al MSA, no obstante, en la serie de pacientes original se describe mayor duración y extensión del bloqueo sensitivo con una inyección superficial al MSA[10]. El mecanismo analgésico también apunta a bloquear la rama lateral de los nervios intercostales en una ubicación más cercana a su emergencia. Comparte la limitación de los bloqueos PECS de no cubrir la rama anterior terminal del nervio intercostal, por lo cual sólo se espera un bloqueo de la pared torácica antero-lateral sin cubrir la línea media, la región mamaria medial y la región torácica posterior medial.

Durante los últimos años ha aumentado el número de estudios controlados randomizados (ECR), fundamentalmente en cirugía mamaria, que comparan el uso de los bloqueos PECS y BPS contra otras estrategias analgésicas, permitiendo un análisis basado en evidencia de mejor calidad.

En el caso del bloqueo PECS I existe un ECR comparando este bloqueo contra placebo en cirugía de mama oncológica (mastectomía y tumorectomía), en el cual no se demostró diferencia en el dolor en la unidad de recuperación a los 30 minutos[11]. La comparación entre PECS I y PECS II en otro ECR evidenció la inferioridad del primero en cuanto a duración de analgesia en mastectomía radical[12]. En suma, el bloqueo PECS I aislado no tiene evidencia que susten- te su uso como técnica analgésica.

El bloqueo PECS II ha sido comparado en ECRs contra analgesia sistémica y BPV con favorables resultados. Un metaanálisis reciente analizó 13 ECRs y un total de 815 pacientes[13], logrando demostrar que el bloqueo PECS II se asocia con un menor consumo de opioides y menor dolor postoperatorio en las primeras 24 horas comparado con analgesia sistémica. En comparación con el BPV, el bloqueo PECS II presenta similar consumo de opioides y control del dolor postoperatorio. Lamentablemente, la magnitud de la diferencia entre ambas comparaciones parece poco significativa clínicamente.

El BPS ha sido evaluado en ECRs para cirugía mamaria y torácica. En comparación con analgesia sistémica Mazzinari et al., demostraron que el BPS disminuye el consumo de morfina en las primeras 24 horas en cirugía mamaria oncológica[14]. No obstante, en el mismo grupo objetivo Gupta et al., evidenciaron la superioridad del BPV sobre el BPS en cuanto a consumo de morfina postoperatorio y tiempo a primera solicitud de analgesia[15]. En cirugía torácica abierta el efecto del BPS fue inferior en lograr disminución de la presión arterial en comparación con AET[16]. Aparentemente, el efecto del BPS es superior a la analgesia sistémica en cirugía videotoracoscópica en términos de calidad de recuperación (score QoR-40) y requerimiento intraoperatorio de remifentanilo[17],[18].

\section{Bloqueo del plano del músculo erector de la columna}

El bloqueo del plano del músculo erector de la 


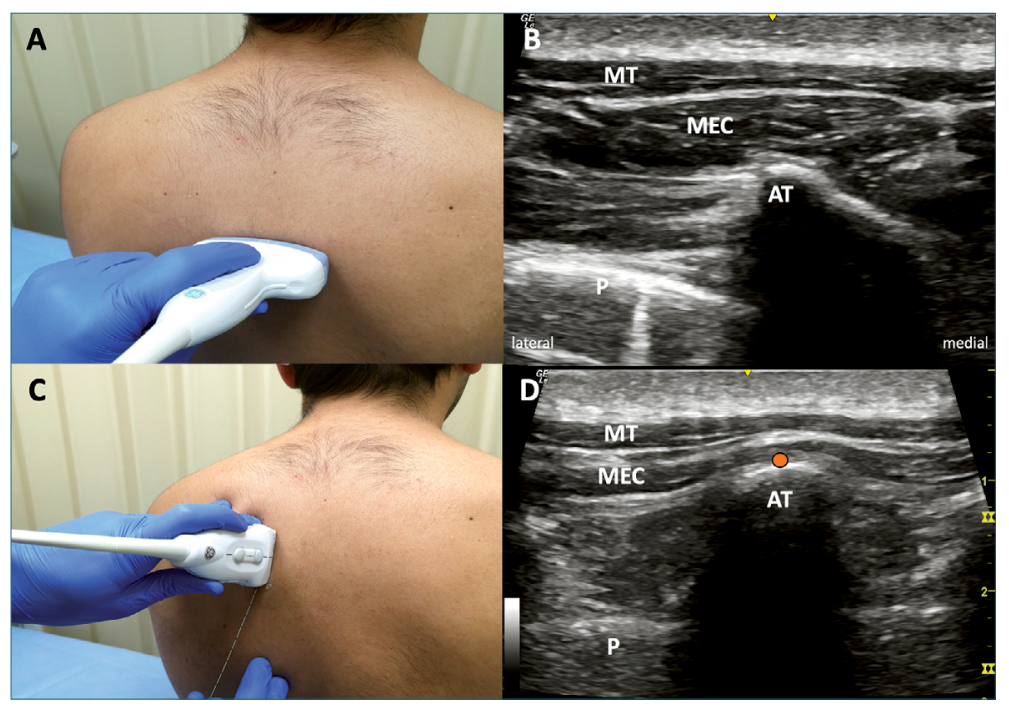

Figura 4. Bloqueo ESP. A: ubicación transversa transductor. B = sonoanatomía en ubicación transversa para localización apófisis transversa. C: ubicación parasagital transductor para bloqueo ESP. D: sonoanatomía ESPB. AT = apófisis transversa, $\mathrm{MEC}=$ músculo erector de la columna, $\mathrm{P}=$ pleura, $\mathrm{TM}=$ músculo trapecio punto naranja $=$ sitio inyección ESP.

columna (en inglés ESPB) es una técnica recientemente descrita por Forero et al., en 2016[19]. El objetivo consiste en inyectar en un plano profundo al músculo erector de la columna y superficial al extremo lateral de la apófisis transversa, posicionando el transductor lineal en posición parasagital (Figuras 1 y 4). En la primera descripción se inyectó AL a nivel de T5 $(20 \mathrm{ml}$ de bupivacaína $0,25 \%$ ) logrando bloqueo sensitivo entre T2 y T9 además de disminución significativa del dolor neuropático[19].

Durante los años siguientes se generó un gran interés en este bloqueo, apareciendo múltiples reportes de su uso como técnica analgésica y anestésica en diversas cirugías. Los ECRs disponibles hasta el momento han analizado su uso en cirugía cardíaca, torácica video-asistida, mamaria, de columna lumbar y abdominal laparoscópica[20]-[30].

En cirugía cardíaca, un ECR comparó el ESPB en bolo más infusión continua realizado a nivel de T6 contra bloqueo epidural en C7-T1, demostrando que ambos alcanzan igual control del dolor durante las primeras 12 horas, sin embargo, los pacientes del grupo ESPB mostraron menores cifras de dolor a las 24, 36 y 48 horas[20]. El estudio carece de registro clínico y no es ciego, por lo cual sus conclusiones deben ser analizadas con cautela. Un segundo ECR evaluó el ESPB contra analgesia sistémica, demostrando que durante las primeras 12 horas el primero es más efectivo en el control del dolor postoperatorio, no obstante, se utilizaron dosis de $A L$ en el límite del máximo sugerido (3 mg/kg de ropivacaína al 0,375\%)[21].

En cirugía mamaria oncológica 2 ECRs han confirmado que el uso de ESPB se relaciona con un menor uso de opioides durante las primeras 24 horas comparado con analgesia sistémica[22],[23]. Sin embargo, en el mismo grupo de pacientes, otro ECR demostró la superioridad del bloqueo PECS II sobre el ESPB en cuanto a consumo de opioides y dolor postoperatorio[24]. En cirugía plástica mamaria otro estudio demostró que el ESPB es superior a la anestesia tumescente para cirugía de reducción mamaria[25].

En cirugía videotoracoscópica ha sido comparado contra analgesia sistémica y BPS. En ambos casos el ESPB fue superior en cuanto a consumo de opioides y dolor postoperatorio[26],[27].

Para cirugía abdominal el ESPB se ha utilizado en colecistectomía laparoscópica, siendo comparado contra analgesia sistémica con resultados favorables[28], y contra bloqueo TAP subcostal. Comparado contra este último un ECR mostró superioridad del ESPB en consumo de analgésicos y dolor postoperatorio[29], mientras que Tulgar et al., no encontraron diferencias entre ambos[30].

El mecanismo por el cual el ESPB ejerce su efecto todavía se encuentra en discusión. Los estudios cadavéricos reportados a la fecha son contradictorios; mientras Ivanusic et al.[31] y Aponte et al.[32], no lograron demostrar difusión significativa del inyectado hacia el espacio paravertebral (EPV) o el ramo ventral, existen otros autores que sí han demostrado difusión a EPV e incluso al espacio epidural[33],[34]. Los ECR analizados tampoco permiten aclarar esta disyuntiva, ya que en ninguno se reporta la evaluación del bloqueo sensitivo. Considerando además que el volumen y masa de droga de anestésicos locales inyectados es considerablemente alto en algunos ECRs, el efecto 
analgésico mediado por absorción sistémica tampoco puede ser soslayado.

La evidencia acumulada a la fecha permite establecer el uso del ESPB como un complemento a la analgesia multimodal en las cirugías descritas previamente, teniendo a su favor su menor incidencia de complicaciones y efectos adversos, además de la menor dificultad técnica reportada. Su rol como alternativa equivalente a las técnicas neuroaxiales y paravertebral no tiene aún respaldo anatómico ni clínico en la literatura.

\section{Bloqueo del plano del músculo transverso del abdomen}

El bloqueo del plano del músculo transverso del abdomen (TAP) es una denominación que actualmente incluye varios abordajes de un mismo plano interfascial. El objetivo final es la inyección de anestésico local en el plano superficial al MTA[3]. El resultado esperado es el bloqueo de los nervios tóraco-abdominales y los nervios ilioinguinal e iliohipogástrico (Figura 2).

La primera descripción del bloqueo TAP corresponde a una técnica guiada por referencias anatómicas y pérdida de resistencia[35]. El punto de inyección sugerido inicialmente por Rafi fue el triángulo de Petit de la pared abdominal, ubicado anterior a la inserción del músculo latísimo del dorso sobre la cresta ilíaca. O’Donnell en 2006 modificó la técnica describiendo el "doble pop", que consistía en avanzar la aguja de bloqueo profundo a la fascia del músculo oblicuo externo (MOE), y posteriormente atravesando la fascia del MOI para inyectar el AL superficial al MTA[36].
El bloqueo TAP guiado por US fue descrito en 2007 por Hebbard[37], con el objetivo de mejorar la precisión de la técnica táctil y aumentar su rendimiento en pacientes obesos o adultos mayores. El transductor lineal debe ubicarse en una orientación transversal sobre la pared abdominal a nivel de la línea medio axilar, entre el reborde costal y la cresta ilíaca. Este abordaje se ha denominado TAP lateral, y la aguja se dirige de anterior a posterior en plano con el US, teniendo como objetivo la inyección de $A L$ entre el MTA y el MOI (Figura 5A y 5B). Estudios clínicos y cadavéricos posteriormente demostraron que la distribución de $\mathrm{AL}$ que se logra con este abordaje suele cubrir solamente los dermatomas de T10 a L1 y no cubre necesariamente la línea media[38]. Esto se explica porque los nervios tóraco-abdominales de T6 a T9 entran al plano del TAP medial al sitio de inyección, además de la naturaleza multisegmentaria de la inervación de la línea media[6]. Por esta razón, se considera que este abordaje es útil para cirugías que involucren la pared abdominal anterior infraumbilical.

El abordaje subcostal guiado por US fue descrito en 2008 también por Hebbard[39], su objetivo primordial es lograr analgesia de la pared abdominal supraumbilical. El transductor se ubica en posición paralela al reborde costal. La aguja se inserta desde el apéndice xifoides de cefálico a caudal y de medial hacia lateral, teniendo como punto objetivo el plano entre el músculo RA y el TA (Figura 5C y 5D). En comparación con TAP lateral, su contraparte subcostal lograría bloquear más dermatomas (3 vs 4) y alcanzar un bloqueo sensitivo más alto (T8 vs T10)[40].

Finalmente, Carney et al., describieron el aborda-

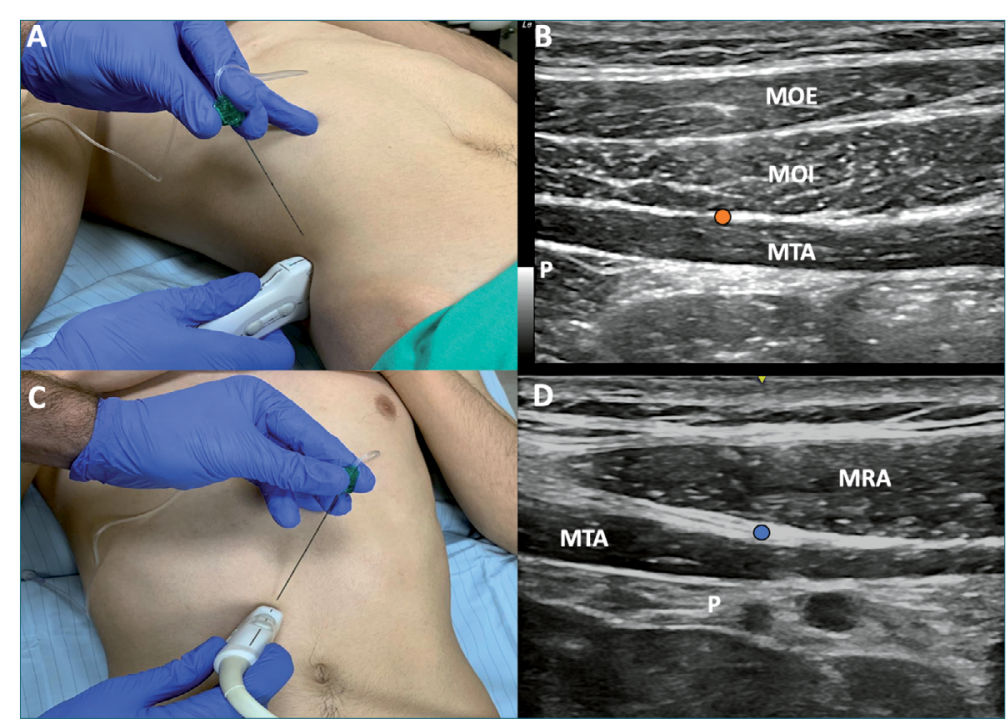

Figura 5. Bloqueos TAP. A: ubicación transductor lineal TAP lateral. B: sonoanatomía bloqueo TAP lateral, punto naranja = sitio inyección TAP lateral. C: ubicación transductor lineal TAP subcostal. D: sonoanatomia bloqueo TAP subcostal. $\mathrm{MOE}=$ músculo oblicuo externo, $\mathrm{MOI}=$ músculo oblicuo interno, MRA = músculo recto del abdomen, MTA = músculo transverso del abdomen, $\mathrm{P}=$ peritoneo. Punto naranja = sitio inyección TAPB lateral, punto azul = sitio inyección TAPB subcostal. 
je posterior guiado por US basado en la descripción del Dr. Rafael Blanco, ubicando el punto de inyección en la intersección del músculo cuadrado lumbar y los músculos abdominales laterales (MOE, MOI y MTA), superficial a la fascia transversalis. En dicho estudio realizado en voluntarios se observó mediante imágenes con resonancia nuclear magnética difusión del $\mathrm{AL}$ hacia el espacio paravertebral entre T5 y T10, además de ausencia de contraste en el plano del TAP[41]. Este abordaje es muy similar en su extensión del bloqueo sensitivo (T5-L1) a la técnica de Rafi, además de compartir la ubicación anatómica del sitio de inyección con el bloqueo del cuadrado lumbar lateral, el cual será analizado más adelante.

La evidencia demuestra en un meta-análisis reciente que el bloqueo TAP guiado por US confiere un beneficio analgésico modesto, referido a una disminución del consumo de morfina a las 6 y 24 horas postoperatorias, en cirugía abdominal con laparotomía, laparoscopía o cesárea[42]. En cirugía pediátrica también existe evidencia de beneficio analgésico, pero sólo en el período perioperatorio precoz (6-8 horas).

En la mayoría de los escenarios clínicos donde se ha intentado utilizar el bloqueo TAP (cirugía abdominal abierta, digestiva laparoscópica, ginecológica, urológica) su beneficio ha sido leve o moderado, en ocasiones no logrando superar la estrategia de analgesia multimodal, o bien siendo inferior a técnicas como la analgesia epidural torácica[3]. Entre las razones que explican este fenómeno se encuentran: heterogeneidad en los abordajes utilizados, efectividad variable de la técnica, necesidad de bloqueo bilateral en cirugías de línea media y ausencia de analgesia visceral.

Los escenarios clínicos donde su uso tiene evidencia clínica de un beneficio significativo son: apendicectomía abierta (TAP lateral o posterior), cesárea en caso de contraindicación para morfina intratecal o indicación de anestesia general (TAP lateral o posterior), cirugía colorrectal abierta si existe contraindicación para analgesia epidural (TAP lateral o subcostal continuo)[38].

En general, se sugiere el uso de concentraciones de bupivacaína entre $0,2 \%$ a $0,25 \%$, con un volumen mínimo de $15 \mathrm{ml}$ por cada lado. Es necesario cautelar no exceder dosis tóxicas en caso de bloqueo bilateral, considerar el uso epinefrina y siempre monitorizar clínicamente por al menos 40 minutos post bloqueo[38].

\section{Bloqueo nervios Ilioinguinal e Iliohipogástrico}

El bloqueo Iliohipogástrico-llioinguinal es una téc- nica recomendada para cirugía inguinal tanto en niños como adultos. Su limitación son aquellas cirugías que requieren analgesia visceral o que comprometen territorio sensitivo del nervio génitofemoral.

Se han descrito varias técnicas guiadas por referencias anatómicas, tomando como referencia un punto medial e inferior a la espina ilíaca anterosuperior (EIAS) y buscando como objetivo el plano entre el MOI y el MTA. La técnica considera uno o dos "pops" al atravesar la aguja los planos musculares y fasciales. Dado que la tasa de falla es entre un $20-40 \%$, es difícil recomendar una técnica específica al respecto[3].

Existen dos abordajes con US. El primero implica ubicar el transductor medial a la EIAS en un plano oblicuo en dirección al ombligo. En dicha posición ambos nervios (Ilioinguinal e lliohipogástrico) suelen encontrarse entre el MOI y MTA (MOE se observa como aponeurosis a este nivel), o bien superficiales al MOI. El otro abordaje consiste en ubicar el transductor superior y posterior a la EIAS, logrando una imagen similar al TAP lateral, compartiendo el mismo punto objetivo de inyección. Si no se identifican los nervios, es posible lograr un bloqueo satisfactorio con 0,1-0-2 ml/kg en el plano descrito[3] (Figura 6).

\section{Bloqueo de la vaina de los rectos}

El objetivo del bloqueo de la vaina de los rectos (BVR) es administrar AL bilateralmente en el plano entre el músculo recto del abdomen y la hoja posterior de la vaina de los rectos. Esto puede lograrse mediante referencias anatómicas y sensación táctil, guiado por US, o bien realizado directamente en el campo quirúrgico por el cirujano. En la técnica ecoguiada el transductor debe situarse en posición transversal sobre la línea media del abdomen, superior al ombligo. En dicha ubicación se identifica la línea alba. Luego se desplaza el transductor hacia lateral hasta encontrar el borde lateral del músculo RA y su vaina. La aguja debe dirigirse desde lateral a medial en plano con el US hasta un punto entre el RA y la hoja posterior de la vaina (Figuras 2 y 6 ). El volumen de $A L$ recomendado es entre $0,1-0,2 \mathrm{ml} / \mathrm{kg}$ (15-20 ml por lado en adultos). El BVR puede ser de utilidad en cirugías que involucren una incisión en línea media, cirugía umbilical y laparoscópica.

Su beneficio analgésico es también moderado, ya que al ser un bloqueo somático carece de analgesia visceral. El análisis de la evidencia acumulada muestra que existe un leve beneficio al comparar el BVR contra infiltración local, más aún cuando los estudios analizados no utilizan analgesia multimodal adecuada[3]. El uso de catéteres para analgesia continua parece ser una alternativa promisoria para cirugía 

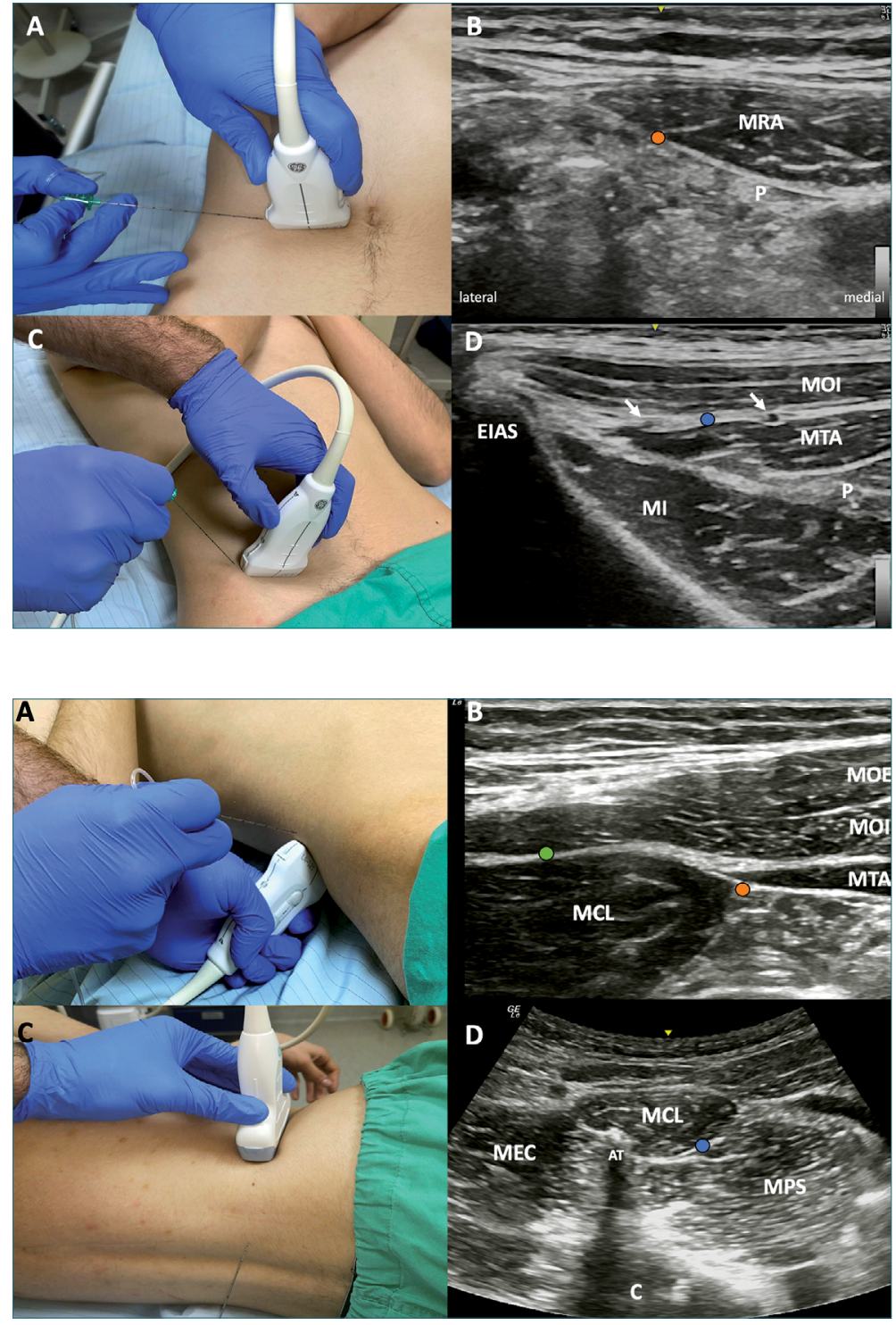

Figura 6. Bloqueos iliohipogástricoilioinguinal y vaina de los rectos. A: ubicación transductor lineal bloqueo vaina de los rectos. B: sonoanatomía bloqueo vaina de los rectos. C: ubicación transuctor lineal bloqueo Ilioinguinal-Iliohipogástrico. D: sonoanatomía bloqueo Ilioinguinal-Iliohipogástrico. EIAS = espina ilíaca anterosuperior, $\mathrm{Ml}=$ músculo ilíaco, $\mathrm{MOE}=$ músculo oblicuo externo, $\mathrm{MOI}=$ músculo oblicuo interno, MRA = músculo recto del abdomen, MTA = músculo transverso del abdomen, $\mathrm{P}=$ peritoneo. Punto naranja= sitio inyección bloqueo vaina de los rectos, punto verde: sitio inyección bloqueo ilioinguinal-iliohipogástrico.

Figura 7. Bloqueo Cuadrado Lumbar. A: ubicación transductor lineal BCL lateral y posterior. B: sonoanatomía BQL lateral y posterior. C: ubicación transductor convexo para $\mathrm{BCL}$ anterior. D: sonoanatomía BCL anterior. AT: apófisis transversa L4, C: cuerpo vertebral L4, $M C L=$ músculo cuadrado lumbar, MEC: músculo erector de la columna, $\mathrm{MOE}=$ músculo oblicuo externo, $\mathrm{MOI}=$ músculo oblicuo interno, MPS: músculo psoas, $\mathrm{MTA}=$ músculo transverso del abdomen, punto naranja = sitio inyección $B C L$ lateral, punto verde = sitio inyección $B C L$ posterior, punto azul = sitio inyección $\mathrm{BCL}$ anterior.

abdominal mayor abierta, no obstante, a la fecha no existen ECR que comparen el BVR contra una técnica neuroaxial.

\section{Bloqueo del cuadrado lumbar}

El bloqueo del cuadrado lumbar (BCL) se origina como una modificación del bloqueo TAP guiado por referencias anatómicas, buscando utilizar como herramienta el US para dirigir la inyección de AL en un plano profundo al $\mathrm{MOI}$ y adyacente al músculo cuadrado lumbar (MCL). Este último tiene una ubicación clave en la pared muscular posterior, en íntima relación con los nervios de la pared abdominal y rodeado por la lámina anterior y media de la fascia tóraco-lumbar. En función del sitio específico de inyección en relación al MCL se han descrito una serie de abordajes, los cuales determinan efectos clínicos disímiles, por lo cual su análisis necesariamente debe ser diferenciado.

La nomenclatura más aceptada e intuitiva ha clasificado al BCL como lateral, posterior o anterior, según cual sea la ubicación final de la aguja en relación al MCL[43]. El abordaje lateral corresponde a la prime- 
ra descripción del bloqueo (BCL tipo 1)[44], ubicando el transductor en una posición transversal al nivel de la cresta ilíaca y cercano a la línea axilar posterior. El punto objetivo de inyección es el borde lateral del $M C L$, profundo al MOI y al MTA. La aguja se inserta en plano desde anterolateral a posteromedial (Figura 2, 7A y 7B). El abordaje posterior (BCL tipo 2) es una inyección sobre la superficie posterior del $\mathrm{MCL}$, profundo al músculo latísimo del dorso y la lámina media de la fascia toracolumbar. La dirección de la aguja es la misma que en el abordaje lateral, sólo que con menor inclinación y buscando una distribución del $A L$ hacia la superficie dorsal del MCL (Figura 2, 7A y 7B) [44]. El abordaje anterior (BCL tipo 3) inicialmente fue descrito como $\mathrm{BCL}$ transmuscular, ya que tiene como objetivo el plano entre el músculo psoas y el $\mathrm{MCL}$, atravesando este último de posterior a anterior para depositar el AL en la superficie ventral del músculo (Figura 2)[45]. Este abordaje requiere posicionar al paciente en decúbito lateral y se sugiere utilizar un transductor convexo de baja frecuencia, ya que se requiere visualizar estructuras más profundas (Figura 7C y 7D).

El BCL logra consistentemente bloqueo sensitivo desde T12-L2 (nervios subcostales, ilioinguinal e iliohipogástrico) independiente del abordaje, ya que el trayecto de los nervios transcurre anterior a la superficie ventral del MCL. La distribución del AL hacia el espacio paravertebral torácico tiene evidencia contradictoria proveniente de estudios cadavéricos y clínicos, sin embargo, se acepta que podría existir difusión del fármaco inyectado hacia dicho espacio y que el nivel cefálico de distribución sería función del nivel vertebral donde se realice el bloqueo[46]. La ventaja que supone la difusión del anestésico hacia el espacio paravertebral es lograr analgesia visceral, aspecto que diferenciaría al $\mathrm{BCL}$ de otros bloqueos de tronco.

Aún existe escasa evidencia proveniente de ECRs en relación con este bloqueo. En cesárea electiva se ha estudiado con resultados favorables en cuanto a disminución del consumo de opioides y dolor postoperatorio versus placebo y TAP[47],[48], no obstante, dicha ventaja desaparece al comparar con morfina intratecal[49],[50]. En cirugía laparoscópica ginecológica ha demostrado disminuir el dolor postoperatorio en las primeras 24 horas[51], aunque recientemente Fujimoto et al., reportó que el uso de $B C L$ no tiene diferencia en la calidad de recuperación versus analgesia sistémica[52]. Para cirugía laparoscopia colorrectal tampoco ha sido superior a placebo[53], ni al uso de infusión continua con lidocaína[54]. En cirugía urológica existe un beneficio en disminuir el dolor y uso de opioides en nefrectomía laparoscópica y nefrolitotomía percutánea[55],[56]. El uso de BCL en colecistectomía laparoscópica también ha sido evaluado contra placebo, aunque sin analgesia multimodal adecuada, logrando mejor analgesia entre las 6 y 24 horas postoperatorias[57].

El volumen de anestésico local sugerido varía entre los distintos autores y reportes, que van desde 0,2 $\mathrm{ml} / \mathrm{kg}$ hasta $30 \mathrm{ml}$ de AL por lado. Se han utilizado concentraciones de bupivacaína y levobupivacaína de 0,125\%-0,5\%[46]. Es necesario siempre considerar el riesgo de toxicidad sistémica, especialmente en caso de bloqueos bilaterales, y adoptar precauciones similares a las descritas previamente (uso de epinefrina y monitorización clínica). Además del riesgo obvio de lesionar estructuras intraabdominales profundas, es importante asumir que el BCL ha sido considerado un bloqueo profundo, por lo cual tiene un riesgo de sangrado que amerita tomar precauciones equiparables a un bloqueo neuroaxial.

\section{Conclusiones}

Los bloqueos de tronco constituyen un avance significativo como alternativa a la técnica neuroaxial. La evolución desde bloqueos de campo hasta técnicas de mayor selectividad ha permitido limitar la masa de anestésicos locales utilizados, manteniendo un adecuado efecto analgésico. El interés actual en el estudio de los bloqueos de tronco será fundamental para esclarecer las indicaciones de cada técnica basado en la mejor evidencia. La comparación de los bloqueos de tronco contra los estándares analgésicos, especialmente en cirugía abierta y con adecuada analgesia multimodal, sigue siendo una tarea pendiente, que por ahora no permite establecer una equivalencia con las técnicas convencionales. 


\section{Referencias}

1. Tran DQ, Van Zundert TC, Aliste J, Engsusophon P, Finlayson RJ. Primary failure of thoracic epidural analgesia in training centers: the invisible elephant? Reg Anesth Pain Med. 2016 May-Jun;41(3):309-13. https://doi.org/10.1097/ AAP.0000000000000394 PMID:27035462

2. Arnuntasupakul $V$, Van Zundert TC, Vijitpavan A, Aliste J, Engsusophon $P$, Leurcharusmee $P$, et al. A randomized comparison between conventional and waveform-confirmed loss of resistance for thoracic epidural blocks. Reg Anesth Pain Med. 2016 May-Jun;41(3):368-73. https://doi.org/10.1097/ AAP.0000000000000369 PMID:26894628

3. Chin KJ, McDonnell JG, Carvalho B, Sharkey A, Pawa A, Gadsden J. Essentials our current understanding: abdominal wall blocks. Reg Anesth Pain Med. 2017 Mar/Apr;42(2):13383. https://doi.org/10.1097/ AAP.0000000000000545 PMID:28085788

4. Rouvière, H. Anatomía Humana Descriptiva, topográfica y funcional, 11a ed. (c2005 Últ. Reimpr. 2006.

5. Hadzic A. Hadzic's Textbook of Regional Anesthesia and Acute Pain Management, Second Edition.

6. Rozen WM, Tran TM, Ashton MW, Barrington MJ, Ivanusic JJ, Taylor Gl. Refining the course of the thoracolumbar nerves: a new understanding of the innervation of the anterior abdominal wall. Clin Anat. 2008 May;21(4):325-33. https:// doi.org/10.1002/ca.20621 PMID:18428988

7. Blanco R. The 'pecs block': a novel technique for provi- ding analgesia after breast surgery. Anaesthesia. 2011 Sep;66(9):847-8. https:// doi.org/10.1111/j.13652044.2011.06838.x PMID:21831090

8. Woodworth GE, Ivie RM, Nelson SM, Walker CM, Maniker RB. Perioperative Breast Analgesia: A Qualitative Review of Anatomy and Regional Techniques. Reg Anesth Pain Med. 2017 Sep/Oct;42(5):60931. https://doi.org/10.1097/ AAP.0000000000000641 PMID:28820803

9. Blanco R, Fajardo M, Parras Maldonado T. Ultrasound description of Pecs II (modified Pecs I): a novel approach to breast surgery. Rev Esp Anestesiol Reanim. 2012 Nov;59(9):4705. https://doi.org/10.1016/j. redar.2012.07.003 PMID:22939099

10. Blanco R, Parras T, McDonnell $J G$, Prats-Galino A. Serratus plane block: a novel ultrasoundguided thoracic wall nerve block. Anaesthesia. 2013 Nov;68(11):1107-13. https:// doi.org/10.1111/anae.12344 PMID:23923989

11. Cros J, Sengès $P$, Kaprelian $S$, Desroches J, Gagnon C, Labrunie A, et al. Pectoral I Block Does Not Improve Postoperative Analgesia After Breast Cancer Surgery: A Randomized, Double-Blind, Dual-Centered Controlled Trial. Reg Anesth Pain Med. 2018 Aug;43(6):596604. https://doi.org/10.1097/ AAP.0000000000000779 PMID:29672368

12. Goswami S, Kundra P, Bhattacharyya J. Pectoral nerve block1 versus modified pectoral nerve block2 for postoperative pain relief in patients undergoing modified radical mastectomy: a randomized clinical trial. $\mathrm{Br} J$ Anaesth. 2017 Oct;119(4):830-
5. https://doi.org/10.1093/bja/ aex201 PMID:29121291

13. Versyck B, van Geffen GJ, Chin KJ. Analgesic efficacy of the Pecs II block: a systematic review and meta-analysis. Anaesthesia. 2019 May;74(5):663-73. https:// doi.org/10.1111/anae.14607 PMID:30957884

14. Mazzinari G, Rovira L, Casasempere A, Ortega J, Cort L, Esparza-Miñana JM, et al. Interfascial block at the serratus muscle plane versus conventional analgesia in breast surgery: a randomized controlled trial. Reg Anesth Pain Med. 2019 Jan;44(1):52-8. https://doi.org/10.1136/rapm2018-000004 PMID:30640653

15. Gupta K, Srikanth K, Girdhar KK, Chan V. Analgesic efficacy of ultrasound-guided paravertebral block versus serratus plane block for modified radical mastectomy: A randomised, controlled trial. Indian J Anaesth. 2017 May;61(5):381-6. https:// doi.org/10.4103/ija.IJA_62_17 PMID:28584346

16. Khalil AE, Abdallah NM, Bashandy GM, Kaddah TA. Ultrasound-Guided Serratus Anterior Plane Block Versus Thoracic Epidural Analgesia for Thoracotomy Pain. J Cardiothorac Vasc Anesth. 2017 Feb;31(1):152-8. https://doi. org/10.1053/j.jvca.2016.08.023 PMID:27939192

17. Kim DH, Oh YJ, Lee JG, Ha D, Chang YJ, Kwak HJ. Efficacy of Ultrasound-Guided Serratus Plane Block on Postoperative Quality of Recovery and Analgesia After Video-Assisted Thoracic Surgery: A Randomized, Triple-Blind, PlaceboControlled Study. Anesth Analg. 2018 Apr;126(4):135361. https://doi.org/10.1213/ ANE.0000000000002779 PMID:29324496

18. Lee J, Kim S. The effects of 
ultrasound-guided serratus plane block, in combination with general anesthesia, on intraoperative opioid consumption, emergence time, and hemodynamic stability during videoassisted thoracoscopic lobectomy: A randomized prospective study. Medicine (Baltimore). 2019 May;98(18):e15385. https://doi.org/10.1097/ MD.0000000000015385 PMID:31045789

19. Forero M, Adhikary SD, Lopez $\mathrm{H}$, Tsui C, Chin KJ. The Erector Spinae Plane Block: A Novel Analgesic Technique in Thoracic Neuropathic Pain. Reg Anesth Pain Med. 2016 Sep-Oct;41(5):621-7. https://doi.org/10.1097/ AAP. 0000000000000451 PMID:27501016

20. Nagaraja PS, Ragavendran S, Singh NG, Asai O, Bhavya G, Manjunath $\mathrm{N}$, et al. Comparison of continuous thoracic epidural analgesia with bilateral erector spinae plane block for perioperative pain management in cardiac surgery. Ann Card Anaesth. 2018 Jul-Sep;21(3):323-7. https://doi.org/10.4103/aca. ACA_16_18 PMID:30052229

21. Krishna SN, Chauhan $S$, Bhoi D, Kaushal B, Hasija S, Sangdup $T$, et al. Bilateral Erector Spinae Plane Block for Acute Post-Surgical Pain in Adult Cardiac Surgical Patients: A Randomized Controlled Trial. J Cardiothorac Vasc Anesth. 2019 Feb;33(2):368-75. https://doi. org/10.1053/j.jvca.2018.05.050 PMID:30055991

22. Gürkan $Y$, Aksu $C$, Kut $A$, Yörükoglu UH. Erector spinae plane block and thoracic paravertebral block for breast surgery compared to IV-morphine: A randomized controlled trial. J Clin Anesth. 2020 Feb;59:848. https://doi.org/10.1016/j. jclinane.2019.06.036
PMID:31280100

23. Singh S, Kumar G, Akhileshwar. Ultrasound-guided erector spinae plane block for postoperative analgesia in modified radical mastectomy: A randomised control study. Indian J Anaesth. 2019 Mar;63(3):200-4. https:// doi.org/10.4103/ija.IJA_758_18 PMID:30988534

24. Altıparmak B, Korkmaz Toker M, Uysal Al, Turan M, Gümüt Demirbilek S. Comparison of the effects of modified pectoral nerve block and erector spinae plane block on postoperative opioid consumption and pain scores of patients after radical mastectomy surgery: A prospective, randomized, controlled trial. J Clin Anesth. 2019 May;54:615. https://doi.org/10.1016/j. jclinane.2018.10.040 PMID:30396100

25. Oksuz G, Bilgen F, Arslan M, Duman Y, Urfalıoglu A, Bilal B. Ultrasound-Guided Bilateral Erector Spinae Block Versus Tumescent Anesthesia for Postoperative Analgesia in Patients Undergoing Reduction Mammoplasty: A Randomized Controlled Study. Aesthetic Plast Surg. 2019 Apr;43(2):291-6. https:// doi.org/10.1007/s00266-0181286-8 PMID:30535555

26. Ciftci $B$, Ekinci $M$, Celik EC, Tukac IC, Bayrak Y, Atalay YO. Efficacy of an Ultrasound-Guided Erector Spinae Plane Block for Postoperative Analgesia Management After Video-Assisted Thoracic Surgery: A Prospective Randomized Study. Journal of Cardiothoracic and Vascular Anesthesia. Elsevier BV; 2019 Apr; Disponible en: http://dx.doi. org/10.1053/j.jvca.2019.04.026

27. Gaballah KM, Soltan WA, Bahgat NM. Ultrasound-Guided Serratus Plane Block Versus Erector Spinae Block for Postoperative Analgesia After Video-
Assisted Thoracoscopy: A Pilot Randomized Controlled Trial. J Cardiothorac Vasc Anesth. 2019 Jul;33(7):1946-53. https://doi. org/10.1053/j.jvca.2019.02.028 PMID:30930141

28. Tulgar S, Kapakli MS, Senturk O, Selvi O, Serifsoy TE, Ozer Z. Evaluation of ultrasound-guided erector spinae plane block for postoperative analgesia in laparoscopic cholecystectomy: A prospective, randomized, controlled clinical trial. J Clin Anesth. 2018 Sep;49:1016. https://doi.org/10.1016/j. jclinane.2018.06.019 PMID:29913392

29. Altıparmak B, Korkmaz Toker M, Uysal Al, Kugçu Y, Gümüt Demirbilek $S$. Ultrasound-guided erector spinae plane block versus oblique subcostal transversus abdominis plane block for postoperative analgesia of adult patients undergoing laparoscopic cholecystectomy: Randomized, controlled trial. J Clin Anesth. 2019 Nov; 57:316. https://doi.org/10.1016/j. jclinane.2019.03.012 PMID:30851501

30. Tulgar S, Kapakli MS, Kose HC, Senturk O, Selvi O, Serifsoy TE, et al. Evaluation of UltrasoundGuided Erector Spinae Plane Block and Oblique Subcostal Transversus Abdominis Plane Block in Laparoscopic Cholecystectomy: Randomized, Controlled, Prospective Study. Anesth Essays Res. 2019 JanMar;13(1):50-6. https://doi. org/10.4103/aer.AER_194_18 PMID:31031480

31. Ivanusic J, Konishi Y, Barrington MJ. A Cadaveric Study Investigating the Mechanism of Action of Erector Spinae Blockade. Reg Anesth Pain Med. 2018 Aug;43(6):56771. https://doi.org/10.1097/ AAP.0000000000000789 
PMID:29746445

32. Aponte $A$, Sala-Blanch $X$, PratsGalino A, Masdeu J, Moreno LA, Sermeus LA. Anatomical evaluation of the extent of spread in the erector spinae plane block: a cadaveric study. Can J Anaesth. 2019 Aug;66(8):886-93. https:// doi.org/10.1007/s12630-01901399-4 PMID:31119551

33. Yang HM, Choi YJ, Kwon HJ, O J, Cho TH, Kim SH. Comparison of injectate spread and nerve involvement between retrolaminar and erector spinae plane blocks in the thoracic region: a cadaveric study. Anaesthesia. 2018 Oct;73(10):1244-50. https:// doi.org/10.1111/anae. 14408 PMID:30113699

34. Adhikary SD, Bernard S, Lopez $\mathrm{H}$, Chin KJ. Erector Spinae Plane Block Versus Retrolaminar Block: A Magnetic Resonance Imaging and Anatomical Study. Reg Anesth Pain Med. 2018 Oct;43(7):75662. https://doi.org/10.1097/ AAP.0000000000000798 PMID:29794943

35. Rafi AN. Abdominal field block: a new approach via the lumbar triangle. Anaesthesia. 2001 Oct;56(10):1024-6. https:// doi.org/10.1046/j.13652044.2001.02279-40.x PMID:11576144

36. O'Donnell BD, McDonnell JG, McShane AJ. The transversus abdominis plane (TAP) block in open retropubic prostatectomy. Reg Anesth Pain Med. 2006 Jan-Feb;31(1):91. https://doi.org/10.1016/j. rapm.2005.10.006 PMID:16418039

37. Hebbard P, Fujiwara $Y$, Shibata $Y$, Royse $C$. Ultrasound-guided transversus abdominis plane (TAP) block. Anaesth Intensive Care. 2007 Aug;35(4):616-7. PMID:18020088

38. Tran DQ, Bravo D, Leurcharus- mee $\mathrm{P}$, Neal JM. Transversus Abdominis Plane Block: A Narrative Review. Anesthesiology. 2019 Nov;131(5):116690. https://doi.org/10.1097/ ALN.0000000000002842 PMID:31283738

39. Hebbard P. Subcostal transversus abdominis plane block under ultrasound guidance. Anesth Analg. 2008 Feb;106(2):6745. https://doi.org/10.1213/ ane.0b013e318161a88f PMID:18227342

40. Lee TH, Barrington MJ, Tran TM, Wong D, Hebbard PD. Comparison of extent of sensory block following posterior and subcostal approaches to ultrasoundguided transversus abdominis plane block. Anaesth Intensive Care. 2010 May;38(3):452-60. https://doi.g/10.1177/031005 7X1003800307 PMID:20514952

41. Carney J, Finnerty O, Rauf J, Bergin D, Laffey JG, Mc Donnell JG. Studies on the spread of local anaesthetic solution in transversus abdominis plane blocks. Anaesthesia. 2011 Nov;66(11):1023-30. https://doi.org/10.1111/ j.1365-2044.2011.06855.x PMID:21851346

42. Baeriswyl M, Kirkham KR, Kern C, Albrecht E. The Analgesic Efficacy of Ultrasound-Guided Transversus Abdominis Plane Block in Adult Patients: A Meta-Analysis. Anesth Analg. 2015 Dec;121(6):1640-54. https://doi.org/10.1213/ ANE.0000000000000967 PMID:26397443

43. El-Boghdadly $\mathrm{K}$, Elsharkawy $\mathrm{H}$, Short A, Chin KJ. Quadratus Lumborum Block Nomenclature and Anatomical Considerations. Reg Anesth Pain Med. 2016 Jul-Aug;41(4):548-9. https://doi.org/10.1097/ AAP.0000000000000411 PMID:27315184
44. Blanco R, McDonnell JG. Optimal point of injection: the quadratus lumborum type I and II blocks. 2013. Available at http:// www.respond2articles.com/ ANA/forums/post1550.aspx. Accessed November 28, 2015.

45. Børglum J, Jensen K, Moriggl B, et al. Ultrasound-Guided Transmuscular Quadratus Lumborum Blockade. BJA Out of Blue eLetters. 2013. Available at http:// bja.oxfordjournals.org/forum/ topic/brjana_el\%3b9919 https:// doi.org/10.1093/bja/el_9919.

46. Elsharkawy H, El-Boghdadly K, Barrington M. Quadratus Lumborum Block: Anatomical Concepts, Mechanisms, and Techniques. Anesthesiology. 2019 Feb;130(2):322-35. https://doi.org/10.1097/ ALN.0000000000002524 PMID:30688787

47. Blanco R, Ansari T, Girgis E. Quadratus lumborum block for postoperative pain after caesarean section: A randomised controlled trial. Eur J Anaesthesiol. 2015 Nov;32(11):812-

8. https://doi.org/10.1097/ EJA.0000000000000299 PMID:26225500

48. Blanco R, Ansari T, Riad W, Shetty N. Quadratus lumborum block versus Transversus abdominis plane block for postoperative pain after cesarean delivery. Reg Anesth Pain Med. 2016 Nov/Dec;41(6):75762. https://doi.org/10.1097/ AAP.0000000000000495 PMID:27755488

49. Tamura T, Yokota S, Ando M, Kubo Y, Nishiwaki K. A triple-blinded randomized trial comparing spinal morphine with posterior quadratus lumborum block after cesarean section. Int J Obstet Anesth. 2019 Nov;40:32-8. https://doi. org/10.1016/j.ijoa.2019.06.008 PMID:31353177 
50. Irwin R, Stanescu S, Buzaianu C, Rademan M, Roddy J, Gormley C, et al. Quadratus lumborum block for analgesia after caesarean section: a randomised controlled trial. Anaesthesia [Internet]. Wiley; 2019 Sep 15;75(1):89-95. Available from: http://dx.doi.org/10.1111/ anae.14852 PMID:31523801

51. Ishio J, Komasawa N, Kido $\mathrm{H}$, Minami T. Evaluation of ultrasound-guided posterior quadratus lumborum block for postoperative analgesia after laparoscopic gynecologic surgery. J Clin Anesth. 2017 Sep;41:14. https://doi.org/10.1016/j. jclinane.2017.05.015 PMID:28802593

52. Fujimoto $H$, Irie $T$, Mihara $T$, Mizuno $Y$, Nomura $T$, Goto $T$. Effect of posterior quadratus lumborum blockade on the quality of recovery after major gynaecological laparoscopic surgery: A randomized contro-
Iled trial. Anaesth Intensive Care. 2019 Mar;47(2):146-51. https:// .g/10.1177/0310057X19838765 PMID:31090440

53. Boulianne $M$, Paquet $P$, Veilleux $R$, Drolet $S$, Meunier A, Frigault $J$, et al. Effects of quadratus lumborum block regional anesthesia on postoperative pain after colorectal resection: a randomized controlled trial. Surgical Endoscopy [Internet]. Springer Science and Business Media LLC; 2019 Oct 22; Available from: http:// dx.doi.org/10.1007/s00464-01907184-0. PMID:31641913

54. Dewinter G, Coppens S, Van de Velde M, D'Hoore A, Wolthuis A, Cuypers E, et al. Quadratus Lumborum Block Versus Perioperative Intravenous Lidocaine for Postoperative Pain Control in Patients Undergoing Laparoscopic Colorectal Surgery: A Prospective, Randomized, Double-blind Controlled Clinical Trial. Ann Surg. 2018 Nov;268(5):769-
75. https://doi.org/10.1097/

SLA.0000000000002888 PMID:30004914

55. Zhu M, Qi Y, He H, Lou J, Pei Q, Mei $Y$. Analgesic effect of the ultrasound-guided subcostal approach to transmuscular quadratus lumborum block in patients undergoing laparoscopic nephrectomy: a randomized controlled trial. BMC Anesthesiol. 2019 Aug;19(1):154. https://doi. org/10.1186/s12871-019-08254 PMID:31412770

56. Dam M, Hansen CK, Poulsen TD, Azawi NH, Wolmarans M, Chan $\mathrm{V}$, et al. Transmuscular quadratus lumborum block for percutaneous nephrolithotomy reduces opioid consumption and speeds ambulation and discharge from hospital: a single centre randomised controlled trial. Br J Anaesth. 2019 Aug;123(2):e350-8. https://doi. org/10.1016/j.bja.2019.04.054 PMID:31153628 